\title{
Características dos atendimentos e satisfação das mães com a assistência prestada na atenção básica a menores de $\mathbf{5}$ anos em Fortaleza, Ceará
}

\author{
Characteristics of consultation and mothers' satisfaction \\ in primary care for children under 5 years of age, Fortaleza, Brazil
}

\author{
Márcia Maria Tavares Machado ${ }^{1}$ \\ Ângela Silvia Sales Lima ${ }^{2}$ \\ José Gomes Bezerra Filho ${ }^{3}$ \\ Maria de Fátima Antero Sousa Machado ${ }^{4}$ \\ Ana Cristina Lindsay ${ }^{5}$ \\ Francismeire Brasileiro Magalhães ${ }^{1}$ \\ Isabelle da Silva Gama ${ }^{1}$ \\ Antonio Jose Ledo Alves da Cunha ${ }^{6}$
}

${ }^{1}$ Departamento de Saúde Comunitária, Faculdade de Medicina, Universidade Federal do Ceará. R. Prof. Costa Mendes 1608/5० andar, Rodolfo Teófilo. 60430-140 Fortaleza CE. marciamachadoce@uol.com.br

${ }^{2}$ Estratégia Saúde da

Família, Redenção, Ceará

${ }^{3}$ Departamento de Saúde

Comunitária, Universidade

Federal do Ceará

${ }^{4}$ Universidade de Fortaleza.

${ }^{5}$ Harvard School of Public

Health.

${ }^{6}$ Reitoria, Universidade

Federal do Rio de Janeiro.

\begin{abstract}
The scope of this study is to describe aspects related to the dimensions of access, the service characteristics and factors associated with satisfaction of mothers in the city of Fortaleza, Ceará. It is an observational, cross-sectional population-based study conducted in 2008 in Fortaleza, Ceará. Families with at least one child under 5 years of age living at home and who were users of the Unified Health System (SUS) were included, The Pearson chi-square test, and when appropriate the Fisher exact test, were used for the statistical associations. The study included 350 families. Most reasons given for the consultation was sickness and on a larger scale, performed by physicians; $2 / 3$ of mothers reported having been satisfied with the last service received by the child. The main factors associated with satisfaction were: the doctors who treated the mother/child called them by name, the service was given on the day that the mother needed and the waiting room of the unit had entertainment. Access to primary care was well evaluated. The satisfaction of users is related to the relationship established with health professionals, however the organization of the service and infrastructure of the units are still considered weak points.
\end{abstract}

Key words Evaluation, Family Health Program, Primary health care, Child health
Resumo Este estudo tem por objetivo descrever aspectos relacionados às dimensões de acesso, características do atendimento e fatores associados à satisfação das mães em Fortaleza, Ceará. Estudo observacional, transversal, de base populacional, realizado em 2008. Foram incluidas famílias que tinham ao menos uma criança menor de 5 anos residindo no domicilio e que fossem usuárias do Sistema Único de Saúde (SUS). Para as associações estatísticas utilizou-se o qui-quadrado de Pearson e quando pertinente o teste exato de Fisher. Participaram do estudo 350 famílias. A maioria dos motivos alegados para a consulta era por doença e, em maior escala, realizada por médicos; dois terços das mães referiram ter ficado satisfeitas com o ultimo atendimento recebido pela criança. Os principais fatores associados a essa satisfação foram: o profissional ter tratado a mãe/criança pelo nome, o atendimento ter ocorrido no dia em que a mãe necessitou e a sala de espera da unidade possuir entretenimento. $O$ acesso à atenção primária foi bem avaliado, sendo considerado como um ponto positivo. A satisfação das usuárias está relacionada com a relação estabelecida com os profissionais de saúde, porém a organização do serviço e a infraestrutura das unidades ainda são considerados pontos frágeis.

Palavras-chave Avaliação, Programa Saúde da Família, Atenção Primária à Saúde, Saúde da criança 
Introdução

No momento em que se discute a reorganização dos serviços de saúde no Brasil, salienta-se a importância da avaliação do acesso a esses serviços, tendo como perspectiva a atenção integral à saúde da criança, que pressupõe, além de uma assistência baseada em aspectos biopsicossociais, a criação de elos entre a população usuária e os serviços ${ }^{1,2}$.

Tais serviços devem ser vistos como um conjunto contínuo e integrado de ações e equipamentos para a promoção, a prevenção e a proteção voltados para a criança e sua família, buscandose a integralidade da abordagem e do atendimento. É preciso, ainda, lembrar que se deve garantir a igualdade neste acesso, sem preconceitos ou privilégios de qualquer espécie. Assim, a igualdade de acesso e a integralidade da atenção são dimensões constitutivas da universalização dos direitos, tarefa fundamental das políticas sociais ${ }^{3,4}$.

A atenção à saúde da criança representa um campo prioritário dentro dos cuidados à saúde das populações. Para que essa se desenvolva de forma mais efetiva e eficiente, além do conhecimento sobre as características relacionadas à morbimortalidade, tais como aspectos biológicos, demográficos e socioeconômicos, é importante salientar o papel que desempenham os serviços e o sistema de saúde ${ }^{5,6}$. Problemas no cumprimento de normas técnicas por parte dos profissionais, no relacionamento entre profissionalpaciente, falta de equipamentos e outros insumos, deficiências na notificação de dados e dificuldades no processo de trabalho dos profissionais, são aspectos decisivos para uma adequada atenção à saúde.

O objeto desta pesquisa se insere no campo da avaliação dos serviços de saúde voltados para crianças menores de cinco anos, tendo como objetivos descrever aspectos relacionados às dimensões de acesso, características do atendimento e fatores associados à satisfação das mães, no município de Fortaleza, Ceará.

\section{Metodologia}

\section{Tipo de estudo}

Estudo observacional, transversal, de base populacional.

\section{Local do estudo e População alvo}

O estudo foi realizado em Fortaleza, capital do estado do Ceará. No período da pesquisa, em 2008, o município contava com 304 equipes de Saúde da Família, correspondendo a uma cobertura de $45 \%$ da população, e encontrava-se dividida em seis regionais administrativas denominadas de Secretarias Executivas Regionais (SER), que funcionavam com um distrito de saúde, educação, meio ambiente, finanças, assistência social e infraestrutura. É a quarta maior cidade do país, com uma população estimada pelo Instituto Brasileiro de Geografia e Estatística (IBGE) para 2007 de 2.431.415 habitantes, dos quais, 234.674 são crianças na faixa etária de 0 a 4 anos completos $^{7}$, população alvo do estudo.

\section{Critérios de Inclusão}

Foram incluídas famílias que residiam em Fortaleza e que tinham ao menos uma criança menor de 5 anos residindo no domicilio no momento da entrevista, que fossem usuárias do Sistema Único de Saúde (SUS) e cujas mães estavam disponíveis para responder ao questionário.

\section{Amostra}

Utilizou-se amostragem por conglomerados em estágios múltiplos considerando-se a divisão de Fortaleza nas seis SER. O numero amostral foi calculado em 350 famílias, sendo esperado em cada bairro que $80 \%$ das mães residentes buscassem o serviço de atenção básica - proporção esta, considerada com margem de erro de $5 \%$, correção para amostragem por conglomerados de $30 \%$ e reposição de perdas de $10 \%$, distribuídas aleatoriamente nos bairros e setores censitários previamente sorteados.

A amostragem seguiu as seguintes etapas: $1^{\mathrm{a}}$ etapa: em cada SER foram sorteados três bairros, totalizando 18 ; $2^{\text {a }}$ etapa: em cada bairro foram sorteados dois setores censitários, totalizando 36 (mediante mapeamento do IBGE); $3^{\text {a }}$ etapa: identificação do domicílio a ser visitado, segundo os critérios de inclusão.

O ponto de partida em cada setor foi escolhido aleatoriamente (quarteirão e domicílio) sendo os quarteirões percorridos em sentido horário, passando-se ao quarteirão seguinte, até completar o total pretendido de 10 residências por setor censitário, que possuíssem crianças com os critérios estabelecidos. Caso não fosse possível completar a amostra esta- 
belecida de 10 crianças em um só setor, seria tomado, complementarmente, o setor vizinho.

\section{Variáveis estudadas}

Sociodemográficas (idade da mãe, estado civil, cor da pele, escolaridade, renda familiar em salários mínimos, possuir televisão a cores). Relacionadas ao acesso (Consulta nos últimos 6 meses; Hospitalização nos últimos 12 meses; Tipo de serviço procurado (Postos/ centros de saúde, Hospital público, Hospital particular); Existe ou não unidade de saúde próximo de casa; Atendimento ocorreu no dia em que precisou; Tempo de espera se houve agendamento $(<10$ dias, 10 a 30 dias, +30 dias); Tempo de espera p/ realização da consulta (Até $30 \mathrm{~min}, 30 \mathrm{~min}$. a 2 horas, +2 horas); Sala de espera possui entretenimento p/ criança; Costuma procurar ajuda do ACS; ACS costuma encaminhar ao PSF; Costuma ser visitada pelo ACS; Frequência da visita (Mensal, a cada 2 meses, a cada 3 meses). Relacionadas ao ultimo atendimento à criança na atenção básica, realizado nos últimos 6 meses a contar da data da entrevista: A mãe ficou satisfeita com a consulta; Motivo da última consulta (Doenças Prevalentes na infância, Rotina, Outros); Profissional que atendeu (Médico, Enfermeiro, Outro); Sabe o nome do profissional; Profissional perguntou motivo da consulta; Profissional tratou mãe/ riança pelo nome; Profissional examinou e deu informações; A mãe compreendeu as informações recebidas.

Em relação à satisfação materna com o último atendimento da criança solicitou-se ainda que as mães lhe atribuíssem uma nota, numa escala de 0 a 10 .

\section{Coleta de dados}

Inicialmente, foi realizado um estudo piloto com 10 famílias para avaliar a compreensão das questões pelas mães e a factibilidade para execução das entrevistas, após o qual o questionário foi revisto.

Os dados foram coletados entre maio e outubro de 2008, utilizando-se um formulário semiestruturado, com questões abertas e fechadas.

Buscou-se inquirir somente a mãe da criança. O pesquisador, ao selecionar a casa, identificou o horário em que a mesma poderia ser entrevistada, podendo retornar até três vezes ao domicílio. Caso não conseguisse, deveria visitar a casa vizinha (a direita) para identificação de novo binômio criança-mãe.
Os formulários foram aplicados por pesquisadores de campo previamente treinados com supervisão durante toda a coleta.

\section{Análise dos dados}

A análise dos dados foi realizada com os softwares EpiInfo 6.04 e STATA 10, construindo-se tabelas de contingência com distribuição de frequência das variáveis estudadas. Procedeu-se a analise bivariada tendo como desfecho a satisfação das mães com o ultimo atendimento prestado à criança. $\mathrm{Na}$ análise multivariada as variáveis que apresentaram associação com o desfecho $(\mathrm{p}<0,20)$ foram inseridas no modelo logístico. Utilizou-se a técnica dos modelos lineares generalizados pela distribuição binomial e função de ligação logit.

Para as associações estatísticas utilizou-se o qui-quadrado de Pearson e quando pertinente o teste exato de Fisher. Foram considerados estatisticamente significativos valores de $\mathrm{p}<0,05$.

\section{Aspectos éticos}

Foi solicitado consentimento das mães para realização das entrevistas e o projeto foi aprovado pelo Comitê de Ética em Pesquisa da Universidade Federal do Ceará.

\section{Resultados}

Participaram do estudo 350 famílias. Dessas, 327 $(93,4 \%)$ não possuíam cobertura de planos de saúde sendo consideradas usuárias exclusivas do SUS. A maioria das mães entrevistadas tinha entre 20-39; aproximadamente 40\% alegaram não ter companheiro. Mais de dois terços eram da cor negra ou parda e tinham, em media, mais de 8 anos de escolaridade. A maioria das famílias $(88,6 \%)$ tinha renda familiar menor que dois salários mínimos. Porem, a grande maioria (98\%) possuía televisão em cores (Tabela 1).

Em relação ao acesso (Tabela 2), a maioria das crianças havia sido consultada nos últimos 6 meses e uma minoria havia sido internada. Para a consulta, a grande maioria havia procurado posto/centro de saúde ou hospital publico. A maioria das mães relatou haver unidade de saúde próxima a sua casa e aproximadamente dois terços referiu que a criança foi atendida quando precisava, com tempo de espera de até duas horas. Metade das unidades de saúde possuía algum entretenimento para a criança. Em relação 


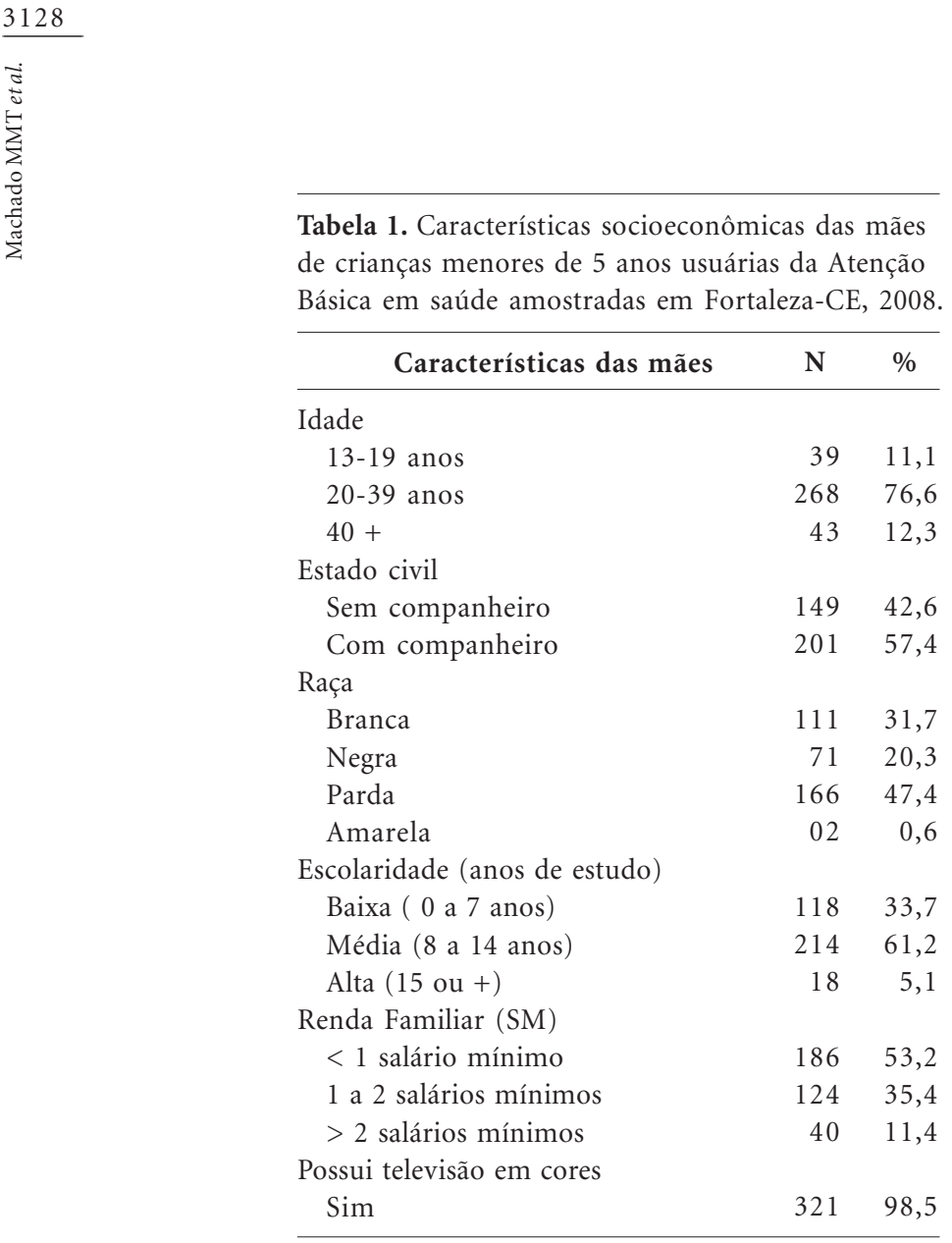

aos agentes de saúde (ACS), a grande maioria das mães alega que não costuma procurar esse profissional e que quando esse tipo de visita domiciliar ocorre, este profissional não costuma encaminhar para a Unidade de Saúde da Família. Além disso, somente metade das mães alega que costuma ser visitada pelo ACS e destas, mais da metade refere ser mensal (Tabela 2).

Em relação ao atendimento (Tabela 3), dois terços das mães referiram ter ficado satisfeitas com o ultimo atendimento recebido pela criança. Quando solicitadas que numa escala de 0 a 10 , atribuíssem uma nota ao atendimento recebido, $15(4,3 \%)$ conferiram valor zero, $68(19,42 \%)$ nota entre 1 e 5 e $263(75,1 \%)$ acima de 6 .

A maioria dos motivos alegados para a consulta era por doença e, em maior escala, realizada por médicos. Metade das mães referiu saber o nome do profissional que atendeu a criança e que na maioria das vezes, este perguntou o motivo da consulta. O profissional tratou a mãe/ criança pelo nome em dois terços das vezes, assim como examinou a criança e deu informações. Na maioria das vezes essas informações foram compreendidas pelas mães (Tabela 3 ).
As seguintes variáveis se mostraram associadas à satisfação materna com a última consulta realizada, na analise bivariada (Tabela 4): "Hospitalização nos últimos 12 meses", "Profissional que atendeu a criança", "Profissional tratou mãe/ criança pelo nome", "Mãe compreendeu as informações recebidas", "Houve atendimento no dia que precisou", "Sala de espera possuía entretenimento $\mathrm{p} /$ criança”. Na analise multivariada, permaneceram associadas à satisfação com a consulta, as seguintes variáveis: "Profissional tratou mãe/criança pelo nome", "Houve atendimento no dia que precisou”, e "Sala de espera possuía entretenimento para a criança” (Tabela 4).

\section{Discussão}

Nosso estudo evidenciou que a maioria das famílias com crianças menores de 5 anos, em Fortaleza (CE), é usuária do SUS e que o acesso aos serviços de saúde é relativamente satisfatório, sendo a maioria dos atendimentos realizados por motivo de doença, com predominância pelo profissional médico. Dois terços das mães referiu estar satisfeita com o atendimento recebido pelas crianças. Os principais fatores associados a essa satisfação foram: o profissional ter tratado a mãe/criança pelo nome, o atendimento ter ocorrido no dia em que a mãe necessitou e a sala de espera da unidade possuir entretenimento para a criança.

Os achados relativos ao processo de atendimento às crianças sugerem uma vinculação positiva dos usuários com os profissionais que lhes atenderam, favorecendo, pois, a participação do usuário durante a prestação do serviço, além da ampliação da eficácia das ações de saúde ${ }^{8,9}$.

$\mathrm{O}$ vínculo dos usuários aos serviços pode ser medido pelo modo como recebem informações relevantes pelos profissionais de saúde ou como apreendem as informações prestadas. Em nosso estudo, verificou-se que $57,7 \%$ das mães entrevistadas conhecem o nome do médico que realizou a última consulta. O ideal seria, no entanto, que todas as mães soubessem o nome do profissional que prestou atendimento a seu filho ${ }^{10}$.

Salienta-se a importância de o cliente ser identificado pelo nome e saber qual o profissional está lhe prestando atendimento. O nome é um dos elementos essenciais em uma relação dialógica, sendo necessário que haja um exercício permanente da menção do nome do paciente durante a consulta. Em se tratando do atendimento à criança, há um significado simbólico em a mãe ouvir do profissional a referência ao nome 
Tabela 2. Utilização do serviço de saúde pelas crianças menores de 5 anos, com trajetória de busca de atendimento e motivos para escolha do serviço amostradas em Fortaleza-CE, 2008.

\begin{tabular}{|c|c|c|c|c|c|}
\hline Características Assistência a saúde & $\mathbf{N}$ & $\%$ & Características Assistência a saúde & $\mathbf{N}$ & $\%$ \\
\hline Consulta nos últimos 6 meses & & & Compreendeu as informações recebidas & & \\
\hline Sim & 319 & 91,1 & Sim & 274 & 78,3 \\
\hline Não & 29 & 8,3 & Não & 23 & 6,60 \\
\hline Hospitalização nos últimos 12 meses & & & Parcialmente & 41 & 11,7 \\
\hline $\operatorname{Sim}$ & 61 & 17,4 & Atendimento no dia que precisou & & \\
\hline Não & 282 & 80,6 & $\operatorname{Sim}$ & 254 & 72,6 \\
\hline Tipo de serviço procurado & & & Não & 85 & 24,3 \\
\hline Postos/ centros de saúde & 238 & 68,0 & Tempo de espera (agendamento) & & \\
\hline Hospital público & 68 & 19,4 & $<10$ dias & 58 & 16,6 \\
\hline Hospital particular & 44 & 12,6 & 10 a 30 dias & 14 & 4,0 \\
\hline Unidade de saúde próximo de casa & & & +30 dias & 5 & 1,4 \\
\hline $\operatorname{Sim}$ & 271 & 77,4 & Tempo de espera p/ realização da consulta & & \\
\hline Não & 54 & 15,4 & Até $30 \mathrm{~min}$. & 127 & 36,3 \\
\hline Motivo da última consulta & & & 30 min. A 2 horas & 131 & 37,4 \\
\hline Doenças prevalentes na infância & 137 & 39,1 & +2 horas & 76 & 21,7 \\
\hline Rotina & 20 & 5,7 & Sala de espera possui entretenimento p/ criança & & \\
\hline Outros & 174 & 49,7 & Sim & 178 & 50,9 \\
\hline Profissional que atendeu & & & Não & 158 & 45,1 \\
\hline Médico & 259 & 74,0 & Procura ajuda do ACS & & \\
\hline Enfermeiro & 60 & 17,1 & $\operatorname{Sim}$ & 33 & 9,4 \\
\hline Outro & 27 & 7,7 & Não & 314 & 89,7 \\
\hline Sabe o nome do profissional & & & ACS encaminha ao PSF & & \\
\hline $\operatorname{Sim}$ & 202 & 57,7 & $\operatorname{Sim}$ & 44 & 12,6 \\
\hline Não & 123 & 35,1 & Não & 301 & 86,0 \\
\hline Profis. Perguntou motivo da consulta & & & Visitada pelo ACS & & \\
\hline Sim & 303 & 86,6 & Sim & 181 & 51,7 \\
\hline Não & 34 & 9,7 & Não & 167 & 47,7 \\
\hline Profis. Tratou mãe/ criança pelo nome & & & Frequência da visita & & \\
\hline Sim & 223 & 63,7 & Mensal & 85 & 24,3 \\
\hline Não & 106 & 30,3 & A cada 2 meses & 53 & 15,1 \\
\hline Profis. Examinou/ deu informações & & & A cada 3 meses & 37 & 10,6 \\
\hline Sim & 253 & 72,3 & & & \\
\hline Não & 84 & 24,0 & & & \\
\hline
\end{tabular}

do filho, preferencialmente, da forma com esta o trata no dia-a-dia. Daí a importância do profissional perguntar e registrar no prontuário o nome normalmente utilizado pela mãe e os demais familiares ${ }^{11}$.

Em relação ao acesso, a grande maioria das crianças havia sido consultada nos últimos 6 meses, ou seja, tiveram suas demandas atendidas. Além disso, o fato da maioria referir ter unidade de saúde perto da residência, que o tempo de agendamento é relativamente curto (menos de 10 dias) e que a espera pela consulta em geral se dá em menos de 2 horas, são indicadores de que acesso não é o maior problema para famílias com crianças menores de 5 anos em Fortaleza.

Importante ressaltar, no entanto, que há espaço para melhoria nessa dimensão da atenção à criança. Por exemplo, nosso estudo evidenciou que a atuação do agente comunitário de saúde pode ser aprimorada.

Assim, possivelmente ocorreria uma diminuição da demanda na unidade de saúde. Além disso, como a maioria das consultas ocorreu por motivo de doença, percebe-se que a ênfase na promoção da saúde e na prevenção de doenças necessita ser bastante ampliado, o que poderia ser realizado em grande parte pelos agentes comunitários de saúde.

A satisfação das mães relacionada ao atendimento prestado à criança descrita nessa investigação conflui para o que foi visto em um estudo referente ao impacto da Estratégia Saúde da Família, no que se trata da importância existente em relação ao nível de comunicação entre o profissi- 
Tabela 3. Associação entre as características de assistência a saúde das crianças menores de 5 anos na atenção básica de Fortaleza e a satisfação das mães, 2008.

\begin{tabular}{|c|c|c|c|c|c|c|}
\hline \multirow[b]{2}{*}{ Características dos domicílios } & \multicolumn{2}{|c|}{ Satisfação } & \multirow{2}{*}{$\begin{array}{l}O R \\
R R\end{array}$} & \multirow[b]{2}{*}{$I C$} & \multirow[b]{2}{*}{$X^{2}$} & \multirow[b]{2}{*}{$P^{*}$} \\
\hline & Sim & Não & & & & \\
\hline \multicolumn{7}{|l|}{ Consulta nos últimos 6 meses } \\
\hline Sim & 202 & 117 & 1.09 & $0.44-2.64$ & 0.04 & 0.84 \\
\hline Não & 16 & 10 & 1.03 & $0.75-1.41$ & & \\
\hline \multicolumn{7}{|l|}{ Hospitalização nos últimos 12 meses } \\
\hline $\operatorname{Sim}$ & 45 & 16 & 1.81 & $0.94-3.54$ & 3.61 & 0.05 \\
\hline Não & 169 & 109 & 1.21 & $1.02-1.45$ & & \\
\hline \multicolumn{7}{|l|}{ Tipo de serviço procurado } \\
\hline Postos/ centros de saúde & 148 & 89 & 0.95 & $0.58-1.57$ & 0.04 & 0.84 \\
\hline Hospitais (público e particular) & 68 & 39 & 0.98 & $0.83-1.17$ & & \\
\hline \multicolumn{7}{|l|}{ Unidade de saúde próximo de casa } \\
\hline Sim & 167 & 103 & 0.72 & $0.36-1.42$ & 1.02 & 0.31 \\
\hline Não & 36 & 16 & 0.89 & $0.73-1.10$ & & \\
\hline \multicolumn{7}{|l|}{ Motivo da última consulta } \\
\hline Doenças & 199 & 112 & 0.96 & $0.33-2.66$ & 0.01 & 0.92 \\
\hline Rotina & 13 & 7 & 0.98 & $0.71-1.37$ & & \\
\hline \multicolumn{7}{|l|}{ Profissional que atendeu } \\
\hline Médico & 172 & 83 & 2.66 & $1.47-4.84$ & 12.25 & 0.00 \\
\hline Enfermeiro & 28 & 36 & 1.54 & $1.15-2.06$ & & \\
\hline \multicolumn{7}{|l|}{ Sabe o nome do profis. } \\
\hline Sim & 139 & 63 & 1.64 & $1.00-2.68$ & 4.33 & 0.03 \\
\hline Não & 70 & 52 & 1.20 & & & \\
\hline \multicolumn{7}{|l|}{ Profis. Tratou mãe/ criança pelo nome } \\
\hline Sim & 166 & 57 & 3.95 & $2.35-6.64$ & 31.86 & 0.00 \\
\hline Não & 45 & 61 & 1.75 & $1.39-2.22$ & & \\
\hline \multicolumn{7}{|l|}{ Profis. Examinou/ deu informações } \\
\hline Sim & 180 & 73 & 9.04 & $3.30-26.06$ & 27.75 & 0.00 \\
\hline Não & 6 & 22 & 3.32 & $1.63-6.78$ & & \\
\hline \multicolumn{7}{|l|}{ Compreendeu as informações recebidas } \\
\hline Sim & 186 & 88 & 4.83 & $1.79-13.50$ & 13.03 & 0.00 \\
\hline Não & 7 & 16 & 2.23 & $1.20-4.16$ & & \\
\hline \multicolumn{7}{|l|}{ Atendimento no dia que precisou } \\
\hline $\operatorname{Sim}$ & 170 & 84 & 1.64 & $0.96-2.78$ & 3.73 & 0.05 \\
\hline Não & 47 & 38 & 1.21 & $0.98-1.49$ & & \\
\hline \multicolumn{7}{|l|}{ Tempo de espera (agendamento) } \\
\hline Até 30 dias & 43 & 29 & 2.22 & $0.28-20.55$ & 0.74 & 0.38 \\
\hline+30 dias & 2 & 3 & 1.49 & $0.50-4.44$ & & \\
\hline Tempo de espera p/ realização da consulta & & & & & & \\
\hline Até 2 horas & 124 & 61 & 2.38 & $1.33-4.26$ & 9.92 & 0.00 \\
\hline+2 horas & 35 & 41 & 1.46 & $1.12-1.89$ & & \\
\hline Sala de espera possui entretenimento $\mathrm{p} / \mathrm{cri}$ & & & & & & \\
\hline $\operatorname{Sim}$ & 134 & 45 & 2.69 & $1.65-4.38$ & 18.04 & 0.00 \\
\hline Não & 82 & 74 & 1.42 & $1.20-1.69$ & & \\
\hline Procura ajuda do ACS & & & & & & \\
\hline Sim & 22 & 11 & 1.20 & $0.53-2.74$ & 0.21 & 0.64 \\
\hline Não & 194 & 116 & 1.07 & $0.82-1.38$ & & \\
\hline ACS encaminha ao PSF & & & & & & \\
\hline Sim & 33 & 10 & 2.09 & $0.95-4.73$ & 3.89 & 0.04 \\
\hline Não & 183 & 116 & 1.25 & $1.04-1.51$ & & \\
\hline Visitada pelo ACS & & & & & & \\
\hline Sim & 110 & 70 & 0.84 & $0.53-1.33$ & 0.63 & 0.42 \\
\hline Não & 107 & 57 & 0.94 & $0.80-1.10$ & & \\
\hline Frequência da visita & & & & & & \\
\hline Mensal & 51 & 33 & 1.03 & $0.54-1.98$ & 0.01 & 0.92 \\
\hline 2 ou mais vezes & 54 & 36 & 1.01 & $0.80-1.29$ & & \\
\hline
\end{tabular}




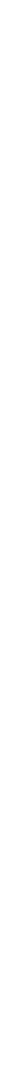

Tabela 4. Satisfação materna com a última consulta realizada.

\begin{tabular}{|c|c|c|c|c|c|c|c|c|}
\hline \multirow[b]{2}{*}{ Condições de acesso } & \multicolumn{2}{|c|}{ Satisfação } & \multirow{2}{*}{$\begin{array}{l}O R \\
R R\end{array}$} & \multirow[b]{2}{*}{$I C$} & \multirow[b]{2}{*}{$P^{*}$} & \multirow{2}{*}{$\begin{array}{c}O R \\
\text { ajustada }\end{array}$} & \multirow[b]{2}{*}{$I C$} & \multirow[b]{2}{*}{$P^{\star}$} \\
\hline & Sim & Não & & & & & & \\
\hline $\begin{array}{l}\text { Hospitalização nos últimos } 12 \text { meses } \\
\text { Sim } \\
\text { Não }\end{array}$ & $\begin{array}{c}16 \\
133\end{array}$ & $\begin{array}{l}45 \\
69\end{array}$ & 0.43 & $0.23-0.87$ & 0.01 & 0.61 & $0.30-1.22$ & 0.16 \\
\hline $\begin{array}{l}\text { Profissional que atendeu } \\
\text { Médico } \\
\text { Enfermeiro }\end{array}$ & $\begin{array}{c}162 \\
97\end{array}$ & $\begin{array}{l}35 \\
30\end{array}$ & 1.43 & $0.82-2.43$ & 0.19 & 1.76 & $0.84-3.70$ & 0.13 \\
\hline $\begin{array}{l}\text { Profis. Tratou mãe/ criança pelo nome } \\
\text { Sim } \\
\text { Não }\end{array}$ & $\begin{array}{c}161 \\
62\end{array}$ & $\begin{array}{l}51 \\
55\end{array}$ & 2.80 & $1.73-4.52$ & 0.00 & 2.69 & $1.60-4.45$ & 0.00 \\
\hline $\begin{array}{l}\text { Compreendeu as informações recebidas } \\
\text { Sim } \\
\text { Não }\end{array}$ & $\begin{array}{c}188 \\
86\end{array}$ & $\begin{array}{l}12 \\
11\end{array}$ & 2.00 & $0.86-4.63$ & 0.10 & 2.04 & $0.71-5.82$ & 0,19 \\
\hline $\begin{array}{l}\text { Atendimento no dia que precisou } \\
\text { Sim } \\
\text { Não }\end{array}$ & $\begin{array}{c}173 \\
44\end{array}$ & $\begin{array}{l}81 \\
41\end{array}$ & 2.00 & $1.17-3.39$ & 0.006 & 2.39 & $1.37-4.18$ & 0.00 \\
\hline $\begin{array}{l}\text { Sala de espera possui entretenimento } \mathrm{p} / \text { criança } \\
\text { Sim } \\
\text { Não }\end{array}$ & $\begin{array}{l}133 \\
82\end{array}$ & $\begin{array}{l}45 \\
76\end{array}$ & 2.74 & $1.69-4.46$ & 0.00 & 2.89 & $1.80-4.93$ & 0.00 \\
\hline
\end{tabular}

onal e o paciente, a compreensão dos problemas pessoais, a forma de tratar o paciente sem discriminação e as explicações sobre as enfermidades ${ }^{12}$.

Um estudo observacional de abordagem quanti-qualitativa ao abordar sobre a satisfação do usuário do Programa Saúde da Família, indicou que a avaliação positiva dos usuários da relação profissional de saúde-usuário confirma a importância da ESF, no estabelecimento de vínculo entre os profissionais de saúde e a população assistida, já que o programa preconiza uma abordagem qualitativa do acolhimento, cuidado à saúde e atendimento humanizado e resolutivo dos problemas de saúde mais frequentes ${ }^{13-16}$.

Os indicadores relacionados ao atendimento prestado à criança, embora não sejam negativos, indicam que muito se pode realizar para aprimorá-los ${ }^{3}$ Aspectos relativos à relação entre o profissional de saúde e a mãe da criança, como por exemplo: perguntar o motivo da consulta, tratar a mãe e a criança pelo nome, examinar a criança e dar informações à mãe e esta compreender as informações, deveriam estar se aproximando a cem por cento das consultas. Entretanto não foi o observado. Nesse sentido, a capacitação continuada dos profissionais de saúde e a supervisão periódica poderiam contribuir para a melhora desses indicadores.
Em relação à satisfação materna com o atendimento de seu filho, fica claro que a maioria esta satisfeita. Este achado é reforçado pelas notas atribuídas pelas mães ao atendimento, numa escala de 0 a 10, tendo a maioria atribuído nota maior que 6. É necessário, no entanto, cautela com esse achado. O possível incremento do acesso aos serviços de saúde somente, pode ter contribuído, em grande parte, pela satisfação com o atendimento ${ }^{17}$. Nossos dados, porém, indicam que a satisfação esteve associada não somente ao acesso, evidenciado pelo atendimento ter ocorrido quando a mãe considerou que precisava.

O acesso é um atributo dos serviços de saúde bastante valorizado pelos usuários. Ao sentiremse acolhidas, as mães procuram além dos seus limites geográficos, serviços receptivos e resolutivos ${ }^{18}$.

É necessário abrir espaços de diálogo com a população, gerando espaços de reflexão e problematização, que possibilitem a construção de uma relação de corresponsabilidade, favorecendo formas mais humanas e efetivas no processo de trabalho em saúde, tanto para os usuários, como para os profissionais ${ }^{14-15}$.

Além dessa variável, a satisfação se mostrou associada ao profissional ter tratado a mãe e a criança pelo nome e a existência de entretenimento 
para a criança na sala de espera. O tratar pelo nome está intrinsecamente relacionado à dimensão da subjetividade do ser humano. Reflete a importância que o profissional, e, por conseguinte a sociedade atribui ao paciente, e que o considera como parte do sistema. Nesse sentido, esta situação esta fortemente relacionada à questão da cidadania. Em relação à existência de entretenimento, pode-se entender de maneira semelhante. Nesse caso, atribuindo-se importância à criança e à sua subjetividade, ou seja, a necessidade de brincar.

Podemos dizer que a sala de espera ameniza o desgaste físico e emocional associado ao tempo de espera por algum atendimento, espera essa que pode gerar ansiedade, angustia, revolta, tensão e comentários negativos em torno do atendimento dos serviços públicos de saúde ${ }^{14-15}$. Daí a necessidade de uma ambiência mais saudável e prazerosa nos serviços de saúde.

Ao analisar a percepção dos profissionais de saúde, gestores e usuários sobre o Programa Saúde da Família, outros estudos também apontaram tal evidência, indicando que os usuários destacam a forma cordial como são tratados pelos membros das equipes, uma questão central para a qualidade da ESF $^{16}$.

Nossos dados devem ser analisados com ressalvas, uma vez que nosso estudo tem limitações. Como foi realizado em 2008, podem ter ocorrido mudanças no sistema local de saúde e nas unidades de saúde de Fortaleza, que tenham alterado para melhor ou para pior nossos resultados. Embora não tenhamos identificado evidências que corroborem essa possibilidade, a mesma deve ser considerada. O caráter populacional de nosso estudo é um aspecto positivo do mesmo. Porem, deve-se ter cautela ao generalizar os dados para toda a população de Fortaleza, uma vez que a amostra, embora probabilística, foi baseada nas Secretarias Executivas Regionais. Além disso, como o estudo foi quantitativo, não foi possível explorar com profundidade aspectos relacionados à satisfação das mães, o que pode ser realizado em outra investigação. Apesar disso, entendemos que as informações obtidas no presente estudo sejam uteis para aprimorar a atenção as crianças menores de 5 anos em Fortaleza.

Em suma, nosso estudo evidenciou que o acesso de crianças menores de 5 anos aos serviços de saúde em Fortaleza é satisfatório e que muito ainda pode ser realizado para melhorar o atendi- mento dessas crianças, em especial na relação profissional de saúde e responsável pela criança.

\section{Conclusões}

Este estudo possibilitou conhecer alguns elementos importantes que estão relacionados ao acesso e à satisfação e/ou à insatisfação do usuário frente à assistência prestada à criança menor de 5 anos, no âmbito da atenção primária. O acesso à atenção primária foi bem avaliado, sendo considerado como um ponto positivo já que esse nível de atenção é a porta de entrada do sistema de saúde.

Outro fator a se considerar é o motivo da procura ao atendimento, destacando-se a doença como foco, onde a promoção da saúde e as estratégias preventivas deveriam ser priorizadas.

A satisfação das usuárias está relacionada com a relação estabelecida com os profissionais de saúde, porém a organização do serviço e a infraestrutura das unidades ainda são consideradas pontos frágeis.

A baixa vinculação dos usuários aos agentes comunitários de saúde denota uma fragilidade na Estratégia Saúde da Família, configurada pela ausência da visita domiciliar na realidade dessas crianças. Apontamos a necessidade da realização de estudos de segmento da Política de Assistência a Saúde da Criança em Fortaleza, ampliando a metodologia dessa pesquisa com a realização de estudos qualitativos buscando compreender a totalidade e as subjetividade das mães para aprofundamento do fenômeno do cuidado das crianças.

Por fim, recomenda-se fortalecer as representações comunitárias nas Câmaras Gestoras Tripartites, nos Conselhos e nos Fundos Municipais de Saúde, como estratégia crucial para a melhoria dos serviços de atenção à saúde da população.

\section{Colaboradores}

MMT Machado, ASS Lima, JG Bezerra Filho, MFAS Machado, AC Lindsay, FB Magalhães, IS Gama e AJLA Cunha participaram igualmente de todas as etapas de elaboração do artigo. 


\section{Referências}

1. Szwarcwald Cl, Damacena GN. Amostras complexas em inquéritos populacionais: planejamento e implicações na análise estatística dos dados. Rev Bras Epidemiol 2008; 11(1):38-45.

2. Victora CG, Aquino EML, do Carmo Leal M, Monteiro CA, Barros FC, Szwarcwald CL. Maternal and child health in Brazil: progress and challenges. Lancet 2011; 377(9780):1863-1876.

3. Victora CG, Matijasevich A, Silveira MF, Santos IS, Barros AJD, Barros FC. Socio-economic and ethnic group inequities in antenatal care quality in the public and private sector in Brazil. Health Policy and Planning 2010; 25(4):253-261.

4. Costa RTE, Bersusa AAS, Saldiva SRDM, Nascimento PR, Venâncio, SI. Integralidade da atenção às doenças cardiovasculares e diabetes mellitus: o papel da regionalização do Sistema Único de Saúde no estado de São Paulo. Rev Bras Epidemiol 2009; 12(2):158-171.

5. Samico I, Hartz ZMA, Felisberto E, Carvalho EF. Atenção à saúde da criança: uma análise do grau de implantação e da satisfação de profissionais e usuários em dois municípios do estado de Pernambuco. Rev Bras Saude Mater Infant 2005; 5(2): 229-240.

6. Fortaleza. Secretaria Municipal de Saúde. Relatório de Gestão - 2006. Fortaleza, 2007. [acessado 2012 out 7]. Disponível em: http://www.sms.fortaleza.ce.gov.br

7. Instituto Brasileiro de Geografia e Estatística (IBGE). População recenseada e estimada, segundo os municípios, 2007. [acessado 2012 out 7]. Disponível em: http://www.ibge.gov.br/home/estatistica/populacao/ contagem2007/contagem_final/tabela1_1_10.pdf

8. Monteiro MM, Figueiredo VP, Machado MFAS. Formulação do vínculo na implantação do Programa Saúde da Família numa unidade básica de saúde. Rev Esc Enferm 2009; 48(2):358-364.

9. Huicho L, Scherpbier RW, Nkowane AM, Victora CG. How much does quality of child care vary between health workers with diff ering durations of training? An observational multicountry study. Lancet 2008; 372(9642):910-916.

10. Machado MMT, Leitão GCM, Holanda FUX de. O conceito de ação comunicativa: uma contribuição para a consulta de enfermagem. Rev Latino-Am Enferm 2005; 13(5):723-728.
11. Machado MMT. Avaliação das atividades dos enfermeiros da Saúde da Família, na atenção à saúde da criança de zero a dois anos em três municípios do Ceará [tese]. Fortaleza (CE): Universidade Federal do Ceará; 2005.

12. Silva AC. O impacto do Programa de Saúde da Família no município de Sobral - Ceará: uma análise da evolução da saúde das crianças menores de cinco anos de idade no período de 1995-2002 [tese]. São Paulo (SP): Universidade de São Paulo; 2003.

13. Cotta RMM, Marques ES, Maia TM, Azeredo CM, Schott M. A satisfação dos usuários do Programa de Saúde da Família: avaliando o cuidado em saúde. Scientia Medica 2005; 15(4):227-234.

14. Oliveira BRG, Collet N, Vieira CS. A humanização na assistência à saúde. Rev Latino-Am Enferm 2006; 14(2):277-284.

15. Rodrigues AD, Dallanora CR, Rosa J, Germani ARM. Sala de espera: um ambiente para efetivar a educação em saúde. Vivências 2009; 5(7):101-106.

16. Ronzani TM, Silva CM. O Programa Saúde da Família segundo profissionais de saúde, gestores e usuários. Cien Saude Colet 2008; 13(1):23-34.

17. Macinko J, Costa MFL. Access to, use of and satisfaction with health services among adults enrolled in Brazil's Family Health Strategy: evidence from the 2008 National Household Survey. Tropical Medicine \& International Health 2012; 17(1):36-42.

18. Victora CG, Barreto ML, do Carmo Leal M, Monteiro CA, Schmidt MI, Paim J, Bastos FI, Almeida C, Bahia L, Travassos C, Reichenheim M, Barros FC. Health conditions and health-policy innovations in Brazil: the way forward. Lancet 2011; 377(9782):2042-2053.

Artigo apresentado em 30/04/2012

Aprovado em 17/07/2012

Versão final apresentada em 30/08/2012 